\title{
Globe
}

Revue internationale d'études québécoises

\section{Jean-François Payette, Introduction critique aux relations internationales du Québec, Québec, Presses de l'Université du Québec, 2009}

\section{David Meren}

Volume 13, numéro 1, 2010

Culture et relations internationales

URI : https://id.erudit.org/iderudit/044651ar

DOI : https://doi.org/10.7202/044651ar

Aller au sommaire du numéro

Éditeur(s)

Globe, Revue internationale d'études québécoises

ISSN

1481-5869 (imprimé)

1923-8231 (numérique)

Découvrir la revue

Citer ce compte rendu

Meren, D. (2010). Compte rendu de [Jean-François Payette, Introduction critique aux relations internationales du Québec, Québec, Presses de l'Université du Québec, 2009]. Globe, 13(1), 188-192. https://doi.org/10.7202/044651ar d'utilisation que vous pouvez consulter en ligne.

https://apropos.erudit.org/fr/usagers/politique-dutilisation/ 
économiques, discursifs, juridiques ou affectifs, ce sont les médiations d'un rapport de don où importe moins la nature de ce qui circule que le sens de l'acte» (p. 229-203). Somme toute, Le cinéma à l'épreuve de la communauté est une contribution significative qui invite à découvrir ou à redécouvrir l'héritage légué par cet organisme qui a permis l'éclosion d'une riche tradition cinématographique.

Mélissa Thériault

Université du Québec à Montréal

\section{Jean-François Payette \\ Introduction critique aux relations internationales du Québec, \\ Québec, Presses de l'Université du Québec, 2009.}

Dans cette Introduction critique aux relations internationales $d u$ Québec, Jean-François Payette a pour objectif de «confronter les réalités pragmatiques des relations internationales du Québec» (p. 32) et de développer un modèle théorique susceptible de fournir aux États subétatiques la base d'une véritable politique étrangère. Selon Payette, il est tout à fait crucial que ces États aient les moyens de se doter d'une politique étrangère dans une conjoncture internationale marquée par la mondialisation et l'intégration régionale.

Issu d'un mémoire de maîtrise, le livre s'ouvre sur un état de la question et la description de l'approche théorique qui servira à analyser l'évolution des relations internationales du Québec. Payette propose ensuite un tour d'horizon de l'histoire de ces relations, suivi d'une évaluation des bases théoriques, légales et politiques de l'action internationale du Québec.

Les lecteurs qui cherchent une analyse critique du contenu des relations internationales du Québec, ou une exploration des activités québécoises dans la sphère non gouvernementale, seront déçus. En effet, sous l'étiquette d' "introduction critique", on découvre vite un appel passionné à la montée en puissance du Québec sur la scène mondiale. Payette critique en fait les bases juridiques et théoriques de l'action internationale du Québec pour mettre en lumière un manque de légitimité et de souveraineté dans le cadre du fédéralisme canadien. L'auteur soutient que les études existantes sur les relations internationales du Québec manquent souvent de précision en ce 
qui concerne leur définition du concept de "politique étrangère»; il constate que seules les entités souveraines peuvent conduire une véritable politique étrangère. En conséquence, Payette propose une approche théorique qui lui permet d'évaluer les activités internationales du Québec (surtout leurs fondements) et de suggérer comment celles-ci pourraient être posées sur une base juridique plus solide. Cela constitue selon Payette un préalable indispensable à l'augmentation des relations internationales du Québec.

Payette maintient que la doctrine Gérin-Lajoie (qui stipule que le Québec possède une capacité internationale en vertu de sa souveraineté dans ses champs de compétence) n'est pas un fondement suffisant pour une véritable politique étrangère car elle se réalise toujours dans le cadre fédéral, imposant ainsi au Québec un statu quo de facto et limitant sa marge de manœuvre. Il décrit la doctrine qui a vu le jour au milieu de la Révolution tranquille comme une sorte de compromis, une manifestation du « rapport structurel entre la souveraineté dominante canadienne et la souveraineté dominée québécoise» (p. 66). Payette reconnaît que la doctrine Gérin-Lajoie permettait au Québec de développer «une pratique internationale» (ce qui ne constitue pas selon lui une politique étrangère), cependant il soutient que la doctrine est sapée fatalement par sa "double allégeance» (p. 22). Cette double allégeance (c'est-à-dire canadienne et québécoise) limite le Québec essentiellement aux champs de compétence prévus dans la Loi constitutionnelle de 1867, et le maintient dans une position subordonnée par rapport à la souveraineté canadienne.

Étant donné les défauts de la doctrine Gérin-Lajoie, Payette propose une "paradiplomatie identitaire» comme fondement de l'action du Québec sur la scène mondiale. Il estime que cette approche peut garantir au Québec une légitimité et une souveraineté internationale accrues et faire cesser l'ingérence du fédéral. Tout en proposant cette alternative, Payette s'éloigne des études précédentes qui ont eu tendance à assimiler une paradiplomatie à la doctrine Gérin-Lajoie. L'auteur imagine la paradiplomatie identitaire qu'il propose comme étant plus assurée et ambitieuse; elle augmenterait les champs d'action du Québec dans le domaine international au-delà de ceux prévus par la doctrine Gérin-Lajoie, en insistant sur les questions de low politics (c'est-à-dire l'économie, le commerce, les affaires sociales, les droits de la personne, l'environnement, etc.). La paradiplomatie identitaire corrigerait également un échec de la doctrine Gérin-Lajoie, en accordant au Québec le droit incontestable de conclure, de ratifier et de modifier les ententes internationales indépendamment d'Ottawa. 
Payette sait qu'il existe plusieurs obstacles politiques et légaux notamment les tensions inéluctables avec Ottawa - à la réalisation d'une paradiplomatie identitaire. Cependant, il n'indique pas avec précision la manière dont il croit que de tels obstacles peuvent être surmontés. Il soutient néanmoins que l'idée d'une paradiplomatie identitaire doit être poursuivie, car elle aboutirait à un accroissement considérable de souveraineté politique pour le Québec par le biais d'un certain nombre d'attributs juridiques, judiciaires et politiques. Payette souligne qu'il ne propose ni une indépendance complète ni une attaque de front contre la souveraineté canadienne, mais plutôt une «micro-souveraineté» (p. 79) qui serait ainsi réalisée par une politique qui consisterait "sans cesse à s'assimiler des fragments d'une souveraineté qui ne lui était pas impartie à la source» (p. 28). En effet, la proposition de Payette revient à la dévolution complète de la compétence en matière d'affaires étrangères (au moins dans des champs spécifiques), ce qui est conforme à l'idée que l'objectif primordial de la paradiplomatie identitaire est "le renforcement ou l'édification de la nation "minoritaire", dans un pays multinational» (p. 28).

Cet ouvrage est parcouru par une insatisfaction évidente manifestée à l'égard de la situation actuelle. Payette exprime son dédain, voire son hostilité, envers le fédéralisme canadien. La doctrine Gérin-Lajoie, et les nombreuses réussites du Québec dans le domaine de l'action internationale, doivent selon l'auteur être minimisées, dans la mesure où elles ont été réalisées dans un cadre où Ottawa a le dernier mot. L'ouvrage passe aussi outre la participation des Québécois au sein du service extérieur du Canada. Payette déplore également la stagnation de la cause souverainiste ainsi que de l'action internationale québécoise. Il déclare qu'une paradiplomatie identitaire donnerait un nouveau souffle aux relations internationales québécoises et mettrait un terme à la «stagnation politique" au Québec (p. 87). En effet, Payette suggère que sa proposition constitue une façon non seulement de préparer la communauté internationale à l'indépendance québécoise, mais également d'obtenir l'aide des membres de cette communauté dans la réalisation de cet objectif.

Cette grogne fondamentale est un atout de l'Introduction critique parce qu' elle incite Payette à remettre en cause un certain nombre d'idées reçues, notamment la valeur de la doctrine Gérin-Lajoie, et à développer dans des termes théoriques et pratiques - une alternative qui permettrait au Québec (au moins en théorie) d'augmenter son action internationale sans pour autant accéder à l'indépendance. Le souci de précision et le mépris du pragmatisme de Payette le poussent à adopter une approche exhaustive: dans 
environ 90 pages, il explore son sujet selon une perspective épistémologique, théorique et politique. Pourtant, cette insatisfaction est également une faiblesse. L'histoire politico-constitutionnelle du Québec et du Canada sur laquelle Payette fonde son argument est assez réductionniste; les rapports perpétuellement antagonistes qu'il décrit lui permettent de présenter la paradiplomatie identitaire comme la seule solution logique et raisonnable.

Létude aurait aussi tiré avantage d'une comparaison plus détaillée du cas québécois avec les autres entités subétatiques (par exemple, la Catalogne, les régions de la Belgique et l'Écosse) auxquelles il fait allusion à plusieurs reprises. Sont-elles vraiment dans une situation supérieure à celle du Québec quant à leurs activités internationales? L'absence de cette comparaison détaillée empêche une évaluation équilibrée des accomplissements de la doctrine Gérin-Lajoie, et l'ouvrage effleure un peu rapidement le rôle innovant du Québec dans les relations internationales. Payette ne mentionne pas, par exemple, la Convention de l'UNESCO pour la protection et la promotion de la diversité des expressions culturelles signée en 2005, qui fut l'occasion d'une forte collaboration entre Québec et Ottawa.

Payette reste également silencieux sur la question du comment. Comment la paradiplomatie identitaire fonctionnerait-elle? Notamment en matière de relations avec le fédéral? L'auteur se contente de faire référence à "une dialectique d'ajustement et d'articulation continuelle avec la souveraineté dominante» (p. 82). Cette situation différerait-elle vraiment de la dynamique actuelle encadrée par la doctrine Gérin-Lajoie? De la même façon, Payette aurait pu explorer les réponses potentielles de la législation internationale et des autres États souverains - surtout ceux composés d'entités subétatiques - au modèle qu'il propose. En effet, les lecteurs sont en droit de se poser (avec plus de scepticisme peut-être) la question que Louise Beaudoin pose dans la préface: "cette nouvelle théorie est-elle opérationnelle?».

En dépit de ces remarques, Introduction critique aux relations internationales $d u$ Québec est une contribution utile et provocante au domaine des relations internationales québécoises. Cet ouvrage examine d'un œil critique les fondements de l'action internationale du Québec, et propose que cette action soit évaluée avec une plus grande rigueur théorique, intellectuelle, légale, et politique. Payette nous donne aussi un avant-goût d'une possible future politique québécoise en matière de relations internationales. C'et important, en raison de l'évolution de la vie politique québécoise pendant la première décennie du XXI ${ }^{\mathrm{e}}$ siècle, des différends 
récurrents entre Québec et Ottawa en matière de relations internationales et des conséquences de la mondialisation.

David Meren

Université Paris IV-Sorbonne/London School of Economics

\section{Anne Trépanier (dir.) \\ La rénovation de l'héritage démocratique. Entre fondation et refondation, Ottawa, Les Presses de l'Université d'Ottawa, 2008.}

Avec le temps, les livres collectifs se sont imposés comme la voie obligée de l'écriture et de la publication universitaires. Préparés à la suite d'un colloque dont ils mûrissent l'idée ou d'un appel de textes lancé pour en tester une, ces livres sont peut-être rarement lus d'une seule traite, et parfois ne sont pas faits pour être lus de la sorte. Les incongruités de construction d'un tel type de livre s'expliquent souvent par le procédé lui-même : un directeur de publication caresse un beau projet autour d'une idée prometteuse et convie une brochette d'auteurs dispersés à la gestation de textes dont il récolte la moisson, un peu comme un pêcheur jetant un filet à l'eau pour voir ce qu'elle contient. Or, d'ordinaire, ce sont les disparates entre les textes réunis qui affaiblissent un livre collectif; dans le cas de l'ouvrage dirigé par Anne Trépanier, qui prend soin d'indiquer qu'il n'est pas un collage, c'est le filet lui-même qui apparaît mal tissé, fait de mailles lâches et drôlement nouées, dans lequel sont pris des textes souvent intelligents et subtils, qui auraient mérité d'être autrement servis.

Le grand filet cousu par Anne Trépanier se déploie à peu près comme suit: la nation et la révolution, porteuses de transcendances radicales et tributaires de l'idée de fondation, sont à dépasser. Armons-nous du concept de refondation, «issue de la conceptualisation de l'idée de fondation, qui s'oppose à l'idée de révolution ", écrit Trépanier, pour l'appliquer à l'histoire canadienne-française devenue québécoise, à l'idée de liberté en général, puis, derechef, à l'histoire, ainsi qu'à l'identité politique, en particulier celles du Canada français hors Québec et des Autochtones. Beau programme en trois parties. Mais de quoi est-il question au juste? De refonder les bases politiques d'une communauté politique, par une action constituante, un moment de grande mobilisation collective, une revivifica- 